\title{
Unexpected Occurrence of Fetal Hemophagocytic Syndrome in a Patient with Hereditary Diffuse Leukoencephalopathy with Spheroids
}

\author{
Yasufumi Kondo, ${ }^{1,2}$, Michiaki Kinoshita1,3*, Takuhiro Yoshida, ${ }^{1,4}$, Hisanori Matoba5, \\ Takeshi Uehara ${ }^{5}$, Meguru Ikeyama ${ }^{6}$, Jun Nakayama ${ }^{6}$, Kunihiro Yoshida ${ }^{7}$, Shu-Ichi Ikeda1 \\ ${ }^{1}$ Department of Medicine (Neurology \& Rheumatology), Shinshu University School of Medicine, \\ Matsumoto, Japan \\ ${ }^{2}$ Department of Neurology, Komoro Kosei General Hospital, Komoro, Japan \\ ${ }^{3}$ Department of Neurology, Suwa Red Cross Hospital, Suwa, Japan \\ ${ }^{4}$ Department of Neurology, Ina Central Hospital, Ina, Japan \\ ${ }^{5}$ Department of Laboratory Medicine, Shinshu University School of Medicine, Matsumoto, Japan \\ ${ }^{6}$ Department of Molecular Pathology, Shinshu University Graduate School of Medicine, Matsumoto, Japan \\ ${ }^{7}$ Department of Brain Disease Research, Shinshu University School of Medicine, Matsumoto, Japan \\ Email: *mkinoshi@shinshu-u.ac.jp
}

Received 30 January 2016; accepted 1 March 2016; published 4 March 2016

Copyright (C) 2016 by authors and Scientific Research Publishing Inc.

This work is licensed under the Creative Commons Attribution International License (CC BY).

http://creativecommons.org/licenses/by/4.0/

(c) (i) Open Access

\section{Abstract}

Colony stimulating factor-1 receptor (CSF1R) plays important roles in the differentiation and proliferation of macrophage and microglia in systemic organs and the brain. A genetic defect in CSF1R causes hereditary diffuse leukoencephalopathy with spheroids (HDLS). HDLS mainly affects the cerebral white matter and shows pre-senile cognitive decline, motor disturbance, and epilepsy. However, systemic manifestations outside the brain have not yet been described in patients with HDLS. Here, we report the case of a 41-year-old man with HDLS carrying the p. K793T mutation in $C S F 1 R$, who unexpectedly died of sepsis and hemophagocytic syndrome shortly after the onset of HDLS. The fetal sequence of sepsis and hemophagocytic syndrome was triggered by enterocolitis. An autopsy revealed that focal inflammation in the intestine had almost resolved. Most strikingly, massive infiltration of cluster of differentiation (CD) 68- and CD163-immunopositive macrophages with hemophagocytosis was observed in the bone marrow, spleen, and liver. Less abundant infiltration of CD68- and CD204-immunopositive macrophages without hemophagocytosis was also seen in the lung and intestine. At present, the pathogenetic link between $C S F 1 R$ mutation and he-

${ }^{*}$ Corresponding author.

How to cite this paper: Kondo, Y., et al. (2016) Unexpected Occurrence of Fetal Hemophagocytic Syndrome in a Patient with Hereditary Diffuse Leukoencephalopathy with Spheroids. Case Reports in Clinical Medicine, 5, 77-84.

http://dx.doi.org/10.4236/crcm.2016.53014 
mophagocytic syndrome in this patient is unclear. Our case, however, clearly shows that even in patients with HDLS, aberrant activation of functional macrophages can be induced under certain conditions in visceral organs.

\title{
Keywords
}

\author{
HDLS (Hereditary Diffuse Leukoencephalopathy with Spheroids), CSF1R \\ (Colony Stimulating Factor-1 Receptor), Enterocolitis, Macrophage, Hemophagocytic Syndrome
}

\section{Introduction}

Hereditary diffuse leukoencephalopathy with spheroids (HDLS) is an autosomal-dominant cerebral white matter disease showing pre-senile cognitive, behavioral, and motor impairment. Mutation in the colony stimulating factor-1 receptor gene $(C S F 1 R)$ is the only causative factor for HDLS identified to date [1]. CSF1R is mainly expressed in innate immune cells such as monocytes, macrophages, dendritic cells, and microglia in the brain, and CSF-1 signal is considered to play a role in differentiation, proliferation, and functioning of these cells [2] [3]. Therefore, it is very likely that the CSF-1 signal is critically important in the maintenance and functioning of the immune system, but to our best knowledge, no patients with HDLS have been reported to develop immunocompromised status. Herein, we report an autopsy case of HDLS in which the patient died of systemic hemophagocytic syndrome triggered by acute enterocolitis approximately 1 year after onset of HDLS.

\section{Case Report}

This case was previously reported by us to carry the p. K793T (c. 2378A > C) mutation in CSF1R [4]. The patient had been healthy until the age of 40 , when he was first noted to have difficulty in handling a cellphone, a baby bed, or a child seat. At the first admission at age 40, he showed mild cognitive impairment (Mini-Mental State Examination [MMSE] score: 23/30, Hasegawa Dementia Scale-Revised [HDS-R] score: 22/30), and depressive and anxious mood. His Frontal Assessment Battery (FAB) score was 10/18, indicating frontal lobe dysfunction. At this time, his general health was good and routine blood examination results were unremarkable. By genetic testing of the patient and his parents, he was confirmed to have HDLS by de novo CSF1R mutation.

Since discharge from our hospital, his anxiety and irritation worsened. He often suggested suicidal ideation to his wife, and attempted to go out of his house repeatedly. One month after discharge, he was hospitalized again (his second admission) because of his psychiatric symptoms. Galantamine $8 \mathrm{mg} /$ day was started for controlling behavioral and psychiatric symptoms, and sodium valproate $400 \mathrm{mg} /$ day and olanzapine $3 \mathrm{mg} /$ day were further added. Then, his anxiety and irritation improved temporarily, and he was discharged from our hospital.

His cognitive function declined rapidly in a few months after his second discharge. Depression and anxiety got worse again, and impulsive and violent behaviors with suicidal ideation often appeared. The dose of galantamine and sodium valproate was increased but it was not effective. Just 1 year after onset of cognitive disorder, he was admitted for the third time to our hospital.

On admission, his general physical condition and laboratory examination results were unremarkable. The scores of MMSE (15/30), HDS-R (14/30), and FAB (4/18) had decreased compared with those from his former admission. Frontal release signs, including preservation and disinhibition, were remarkable. Although he was able to walk without any assistance, some of the daily life activities including bathing, clothing, and brushing of teeth were disturbed. Compared with magnetic resonance (MR) images at his second hospitalization 4 months ago, atrophy in the cerebral cortex and corpus callosum progressed (Evans index: from 0.328 to 0.349; corpus callosum index: from 0.215 to 0.183) [5]. Single photon emission computed tomography (SPECT) showed a decrease in the blood flow especially in the bilateral frontal and parietal lobes.

Repetitive and impulsive behaviors, probably due to severe irritation and disinhibition, were prominent. Thus, olanzapine was eventually increased to $20 \mathrm{mg} /$ day and levomepromazine $5 \mathrm{mg} /$ day was added. Thereafter, his psychiatric and behavioral symptoms reduced. Instead, drug-induced parkinsonism including bradykinesia, brachybasia, and frozen gait appeared. Furthermore, he developed excessive sweating and hyperthermia (body temperature, $38^{\circ} \mathrm{C}$ ) with hyper-CK (creatine kinase)-emia (maximum value: $7667 \mathrm{IU} / \mathrm{L}$ ). The level of serum 
C-reactive protein (CRP) was not increased at that point. Olanzapine and levomepromazine were stopped. Fluid transfusion, dantrolene sodium, and bromocriptine mesylate were started. Although hyperthermia and hyperCK-emia were favorably improved in a few days, constipation and appetite loss occurred and continued thereafter. He did not have abdominal pain and any other symptoms. He admitted to be doing well with no trouble, and continued the physical and occupational rehabilitation as usual.

Unexpected high fever of $42^{\circ} \mathrm{C}$ with severe diarrhea and shivering occurred suddenly at the 26th day from his most recent hospital admission (day 1 in Figure 1). Escherichia coli (E. coli) harboring extended spectrum beta lactamase (ESBL) were detected in stool and blood cultures, highly suggesting that the intestinal infection expanded to sepsis. Contrary to our expectations, the intensive combination therapy of several antibiotics and immunoglobulin was ineffective (Figure 1). The same resistant bacteria were detected continuously in a series of blood cultures. Despite repeated transfusion of red blood cells (RBC) and platelets (Plt), their counts gradually decreased, while that of white blood cells (WBC) increased (maximum: 33,430/ $\mu \mathrm{L}$, Table 1). Serum CRP (32.74 mg/dL) and ferritin (1631 ng/mL) levels also increased. At day 5 after onset of high fever with severe diarrhea, polymyxin B-immobilized fiber column (PMX)-direct hemoperfusion was done to absorb the endotoxins in the blood, but failed to improve his condition. Bone marrow examination at day 10 showed a small amount of hemophagocytic macrophages. On the 18th day, the number of WBC suddenly decreased to $780 / \mu \mathrm{L}$, and he died on the same day at the age of 41. Retrospectively, the total duration of HDLS was approximately 13 months and that from onset of intestinal infection to death was 18 days.

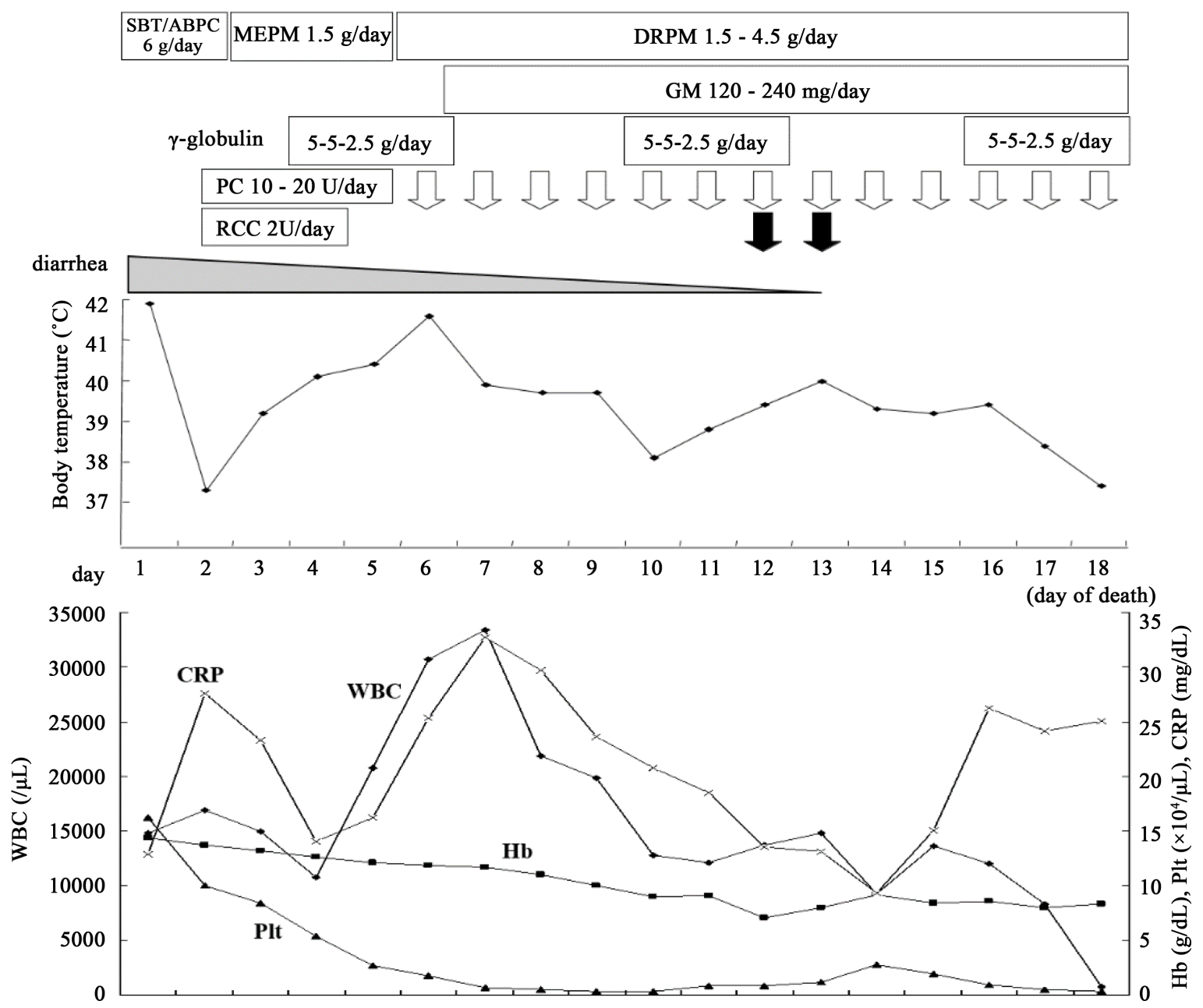

Figure 1. Clinical course. Despite repeated platelet transfusions, the platelet count never recovered. The patient died on day 18 due to septic shock and hemophagocytic syndrome. Abbreviations. SBT/ABPC: sulbactam/ampicillin, MEPM: meropenem, DRPM: doripenem, GM: gentamicin, PC: platelet concentrates, RCC: red cell concentrates. 
Table 1. Serial laboratory findings.

\begin{tabular}{|c|c|c|c|c|c|c|c|}
\hline Day & $1^{*}$ & 5 & 7 & 8 & 15 & 18 & Reference values \\
\hline \multicolumn{8}{|c|}{ Blood biochemistry } \\
\hline $\mathrm{TP}$ & 7.5 & 5.7 & 5.2 & 5.0 & 5.1 & 5.3 & $6.5-8.0 \mathrm{~g} / \mathrm{dL}$ \\
\hline ALB & 4.4 & 2.9 & 2.3 & 2.1 & 2.3 & 2.1 & $4.0-5.0 \mathrm{~g} / \mathrm{dL}$ \\
\hline BUN & 18 & 18 & 41 & 55 & 25 & 51 & $8-21 \mathrm{mg} / \mathrm{dL}$ \\
\hline Cre & 0.82 & 0.77 & 1.08 & 1.09 & 0.72 & 0.93 & $0.63-1.05 \mathrm{mg} / \mathrm{dL}$ \\
\hline AST & 40 & 51 & 93 & 84 & 28 & 26 & $11-28 \mathrm{U} / \mathrm{L}$ \\
\hline ALT & 55 & 33 & 34 & 32 & 30 & 22 & $9-36 \mathrm{U} / \mathrm{L}$ \\
\hline LDH & 324 & 387 & 395 & 447 & 282 & 338 & $120-230 \mathrm{U} / \mathrm{L}$ \\
\hline CK & 277 & 665 & 581 & 336 & 100 & 19 & $43-272 \mathrm{U} / \mathrm{L}$ \\
\hline CRP & 12.88 & 16.26 & 32.74 & 29.73 & 15.08 & 25.09 & $<0.10 \mathrm{mg} / \mathrm{dL}$ \\
\hline PCT & 18.57 & $\mathrm{NE}$ & $\mathrm{NE}$ & 146.61 & $\mathrm{NE}$ & $\mathrm{NE}$ & $<0.5 \mathrm{ng} / \mathrm{mL}$ \\
\hline Ferritin & $\mathrm{NE}$ & 909 & NE & 1631 & 647 & 877 & $25-280 \mathrm{ng} / \mathrm{mL}$ \\
\hline Endotoxin & $\mathrm{NE}$ & 6.78 & $\mathrm{NE}$ & 10.43 & 5.20 & $\mathrm{NE}$ & $<5 \mathrm{pg} / \mathrm{mL}$ \\
\hline \multicolumn{8}{|c|}{ Blood cell count } \\
\hline WBC & 14.81 & 20.75 & 33.43 & 21.90 & 13.61 & 0.78 & $2.97-9.13 \times 10^{3} / \mu \mathrm{L}$ \\
\hline $\mathrm{RBC}$ & 4.66 & 4.02 & 3.87 & 3.65 & 2.78 & 2.83 & $4.14-5.63 \times 10^{6} / \mu \mathrm{L}$ \\
\hline $\mathrm{Hb}$ & 14.4 & 12.1 & 11.7 & 11.0 & 8.4 & 8.3 & $12.9-17.4 \mathrm{~g} / \mathrm{dL}$ \\
\hline Hct & 42.5 & 36.0 & 33.8 & 32.1 & 24.7 & 25 & $38.6 \%-50.9 \%$ \\
\hline PLT & 16.2 & 2.7 & 0.7 & 0.5 & 1.9 & 0.3 & $14.3-33.3 \times 10^{4} / \mu \mathrm{L}$ \\
\hline \multicolumn{8}{|c|}{ Coagulation test } \\
\hline PT & $\mathrm{NE}$ & 13.5 & 13.0 & 12.7 & 13.8 & 13.7 & normal control $\pm 10 \%$ \\
\hline APTT & $\mathrm{NE}$ & 34.5 & 37.9 & 36.1 & 30.0 & 32.8 & $23.0-38.0 \mathrm{sec}$ \\
\hline FIBG & $\mathrm{NE}$ & 487.3 & 589.9 & 527.6 & 270.9 & 302.7 & $180-350 \mathrm{mg} / \mathrm{dL}$ \\
\hline AT-III & $\mathrm{NE}$ & 71.0 & 78.4 & 81.5 & 79.4 & 72.0 & $80 \%-120 \%$ \\
\hline FDP-DD & NE & 2.4 & 2.7 & 2.2 & 4.4 & 4.4 & $\leq 1.0 \mu \mathrm{g} / \mathrm{mL}$ \\
\hline
\end{tabular}

*day1 indicates the day of onset of fever and diarrhea; PCT: procalcitonin; NE: not examined.

\section{Pathological Findings}

Autopsy was performed $1.5 \mathrm{~h}$ after death. Macroscopic findings are shown in Figure 2. The liver weighed 2860 $\mathrm{g}$ (including gall bladder). The spleen weighed $285 \mathrm{~g}$. The right and left lungs weighed $970 \mathrm{~g}$ and $735 \mathrm{~g}$, respectively. The intestinal mucosa looked almost normal macroscopically.

Microscopically, the main pathological lesions were observed in the bone marrow, spleen, and hepatic sinusoids, where a large amount of crowding macrophages or Kupffer cells with hemophagocytosis was seen. Representative histopathology in these tissues is shown in Figure 3.

Immunohistochemical expression of CD68, CD163, and CD204 on phagocytic cells is presented in Figure 4. Phagocytic cells positive for a pan-macrophage marker CD68 were found in various tissues irrespective of hemophagocytosis. However, in tissues where hemophagocytosis was conspicuous such as the bone marrow, spleen, and liver, CD68-positive macrophages or Kupffer cells were also positive for CD163 rather than CD204. In contrast, CD68-positive macrophages in the lung and small intestine, without involvement of hemophagocytosis, were largely positive for CD204 rather than CD163 (Table 2).

Taken together, the main pathological diagnosis was hemophagocytic syndrome. 

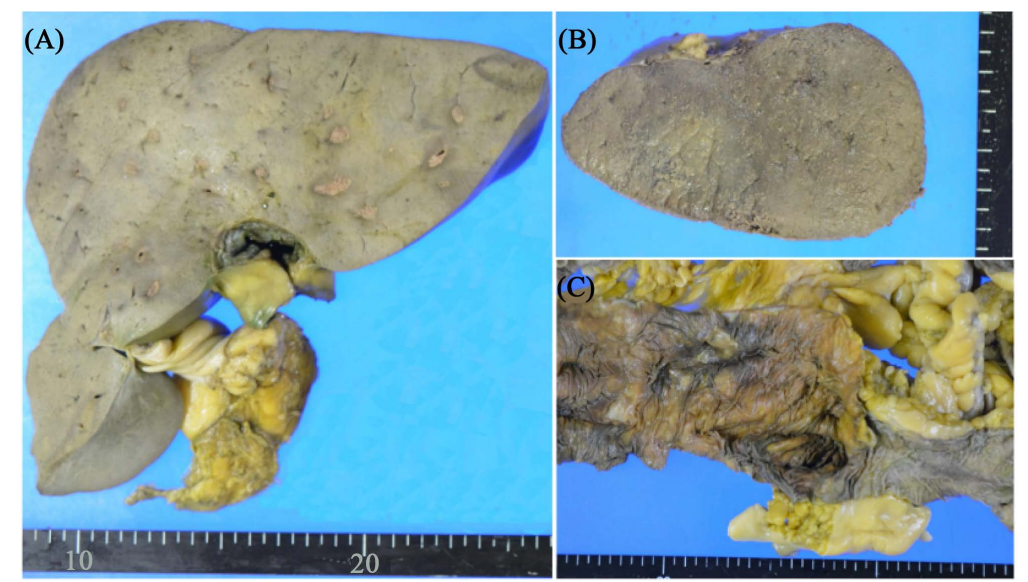

Figure 2. Macroscopic findings. (A) The cut surface of the liver looks yellowish, probably due to a huge accumulation of macrophages; (B) The section of the spleen is reddish and muddy, and the structures including follicles and trabeculae are disrupted and indistinct; (C) The mucosal folds of the jejunum are almost preserved.

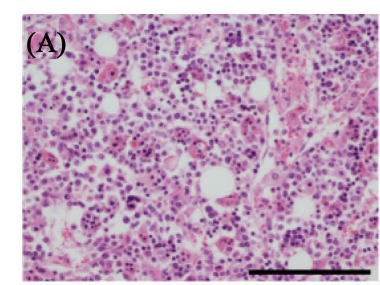

(D)

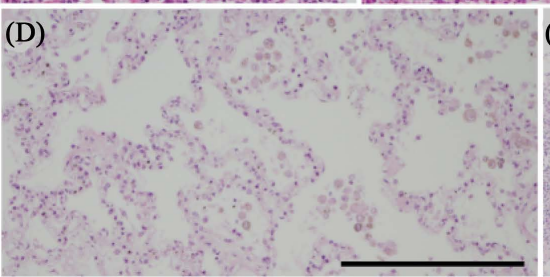

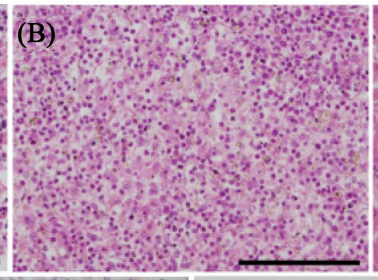

(E)

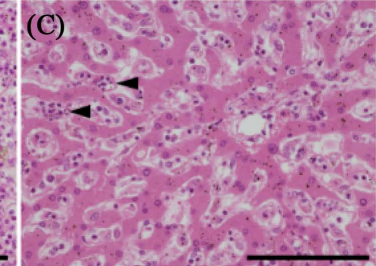

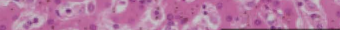

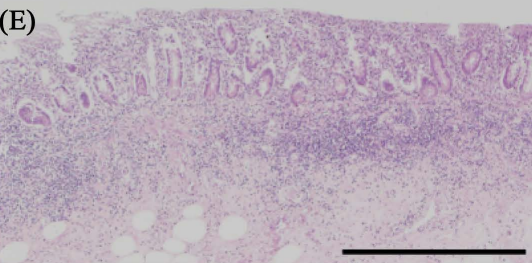

Figure 3. Microscopic findings of various tissues involved. (A) The bone marrow shows marked increased cell numbers including macrophages. Erythroblasts are mainly englobed by hemophagocytic macrophages; (B) In the spleen, white pulp is diminished and red pulp is relatively conspicuous. These findings are compatible with infectious spleen. There is a large amount of macrophages phagocytizing red blood cells and neutrophils; (C) In the liver, Kupffer cells phagocytizing neutrophils (indicated by arrowheads) are evident in sinusoids; (D) In the lungs, edema surrounding the bronchus, interlobular septum, and vessels is noticeable. Alveolar macrophages are relatively small and phagocytosis is not observed. These cells are regarded as tissue-resident macrophages; (E) In the small intestine, a small amount of shedding and bleeding of mucosa is seen, but the mucosa is almost preserved. There are a small number of macrophages, which seem to be non-phagocytic, tissue-resident macrophages in the mucosa. Large phagocytic macrophages are seen only in the mucosal vessels. There is no infiltration of neutrophils. Bars indicate $100 \mu \mathrm{m}((\mathrm{A})-(\mathrm{C})), 200 \mu \mathrm{m}$ (D) and $500 \mu \mathrm{m}$ (E).

Table 2. Semiquantitative evaluation of the immunohistochemistry samples.

\begin{tabular}{cccccc}
\hline & Bone marrow & Spleen & Liver & Lung & Small intestinal mucosa \\
\hline Hemophagocytosis & present & present & present & absent \\
CD68 & +++ & +++ & +++ & +++ & ++ \\
CD163 & +++ & ++ & ++++ & ++ \\
CD204 & ++ & - & ++ & ++ \\
\hline
\end{tabular}

+++: severe, ++: moderate, +: mild, -: absent. 


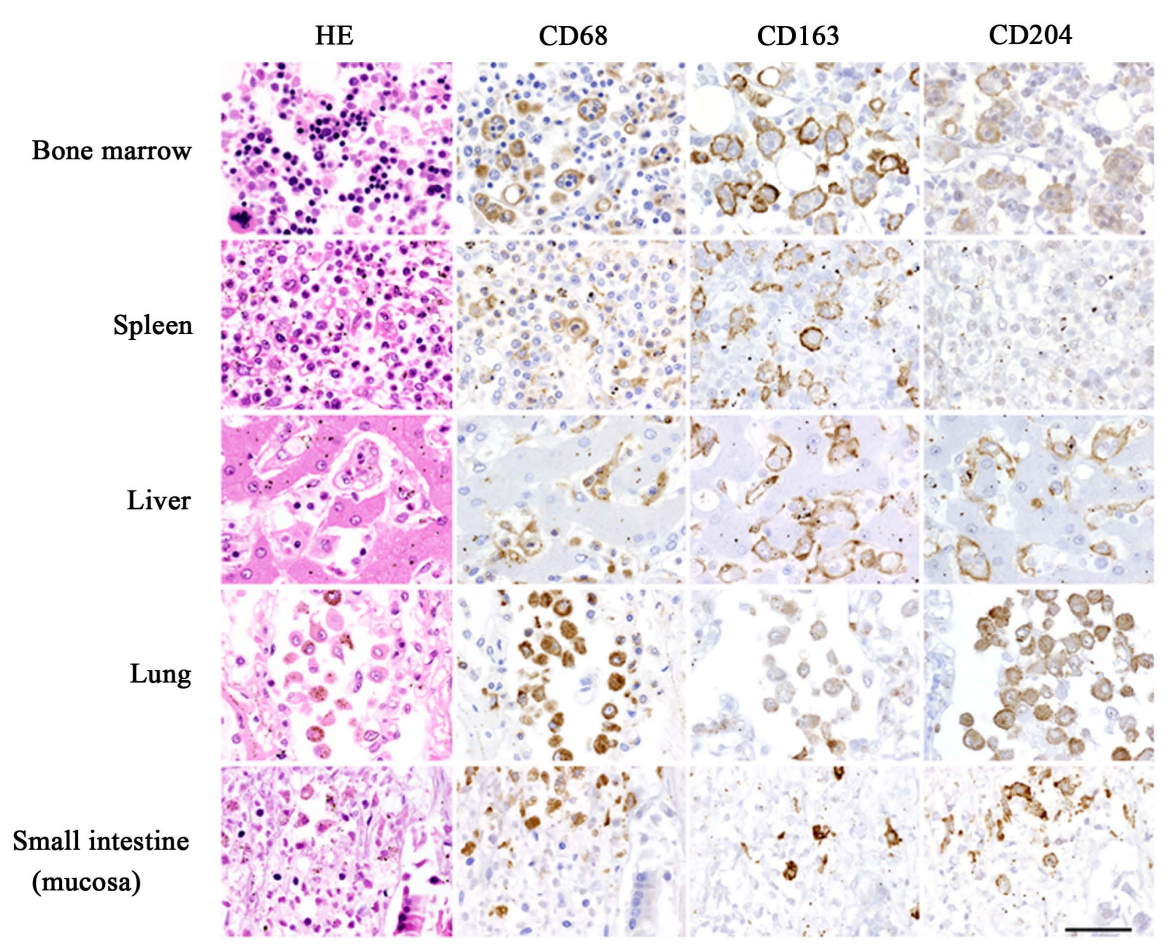

Figure 4. Immunohistochemical findings of infiltrating phagocytic cells. CD68-positive cells are found in the bone marrow, spleen, liver, lung, and small intestinal mucosa. Notably, in tissues showing marked hemophagocytosis, CD68-positive macrophages in bone marrow and spleen as well as CD68-positive Kupffer cells in liver are also largely positive for CD163 rather than CD204. On the other hand, in tissues without involvement of hemophagocytosis, CD68-positive alveolar macrophages in the lung and macrophages in the small intestinal mucosa are also positive for CD204 rather than CD163. Primary antibodies used for immunohistochemistry are monoclonal anti-CD68 (clone KP1, Dako, Glostrup, Denmark), anti-CD163 (clone AM-3K, TransGenic, Kobe, Japan), and anti-CD204 (clone SRA-E5, TransGenic, Kobe, Japan) antibodies. Histofine Simple Stain MAX-PO(M) (Nichrei Bioscience, Tokyo, Japan) was used for the secondary antibody. Bar indicates $50 \mu \mathrm{m}$.

\section{Discussion}

The most striking finding in the present case is that the patient died of sepsis and hemophagocytic syndrome with systemic infiltration of macrophages in the early stage of HDLS. His clinical course was extraordinary for HDLS because excessive proliferation of macrophages in systemic organs has never been described in HDLS. We considered enterocolitis as a trigger of sepsis clinically, but the intestinal mucosa looked well preserved at autopsy. Therefore, we judged that the enterocolitis was cured locally, but the subsequent sepsis and hemophagocytic syndrome with massive infiltration of macrophages were not controlled in this patient.

Macrophages are roughly classified into two different subtypes: classically activated (M1)-type and alternatively activated (M2)-type macrophages [6]. M1-type macrophages are differentiated by granulocyte macrophage-colony-stimulating factor (GM-CSF) and promote inflammation. On the other hand, M2-type macrophages, differentiated by macrophage-colony stimulating factor (M-CSF: CSF-1), contribute to suppression of inflammation and repair of injured tissues [7] [8]. CD68 is regarded as a pan-macrophage marker expressed on monocytes, macrophages, Kupffer cells, and microglia [9] [10]. Both CD163 and CD204 are expressed on M2 macrophages, and are generally regarded as M2-macrophage markers [11] [12]. In addition, CD163-positive macrophage is activated in the bone marrow and spleen in the case of sepsis or hemophagocytic syndrome [13]-[15]. Conversely, CD204 is expressed on the tissue-resident macrophages including alveolar and intestinal macrophages, and these cells maintain the local immune system [16] [17]. These observations were faithfully confirmed in our patient, that is, macrophages in the bone marrow, spleen, and liver, where active hemophagocytosis was observed, were largely positive for CD163 rather than CD204. In contrast, macrophages were 
CD204 predominant in the lung and small intestine, where hemophagocytosis was rarely seen.

We have to consider how the CSF1R mutation is linked with fetal macrophage proliferation in the present case. CSF1R function is generally considered indispensable for the differentiation and proliferation of macrophages [7] [8]. Indeed, it has been reported that macrophages are severely depleted in number or almost absent and their phagocytic functions are profoundly damaged in M-CSF-deficient osteopetrotic (Csf $1^{\text {op/op }}$ ) mice, or CSF1R-disrupted $\left(\mathrm{Csf1}^{-1}\right)$ mice [18]-[20]. Therefore, it seems unlikely that the dysfunction in CSF1R induces over-proliferation and activation of macrophages.

On the other hand, it is also reported that Csf1 ${ }^{\mathrm{op} / \mathrm{op}}$ mice develop colitis and sepsis easily and severely [21]. This study argues that CSF-1 dysfunction may disturb IL-10 mediated regulation of the immune defense system against enterocolitis. This might be a possible explanation for intractable enterocolitis in this patient. Because HDLS is considered to result from haploinsufficiency of CSF1R [22], the pathological features in Csf $1^{\text {op/op }}$ mice could reproduce those of HDLS partly. However, it needs to be kept in mind that not only CSF-1, but also IL-34 can be the ligand for CSF1R.

At present, there is no conclusive proof that $C S F 1 R$ mutation is causative of fetal hemophagocytic syndrome. In fact, clinical and pathological studies on HDLS have been confined to the brain, but not to systemic organs to date. The co-occurrence of HDLS and hemophagocytic syndrome may be a mere coincidence in our patient. Based on studies on animal models described above, however, the possibility cannot be excluded that $C S F 1 R$ mutations may be involved in the dysregulation of macrophages in systemic organs. Therefore, we hope that this case report will enhance the general attention to the pathophysiology of systemic organs in patients with HDLS.

\section{Conclusion}

We here report an autopsy case of a patient with HDLS who died of systemic hemophagocytic syndrome shortly after onset of HDLS. The present case clearly demonstrates that even in patients with HDLS, uncontrollable proliferation of hemophagocytic macrophages can take place in some specific, but undefined conditions.

\section{Acknowledgements}

This work was supported by Grants-in-Aid from the Research Committee for hereditary cerebral small vessel disease and associated disorders, the Ministry of Health, Labor and Welfare of Japan (Dr. K. Yoshida).

\section{Study Ethics Approval}

This study was conducted with the approval of the Ethical Committee of Shinshu University School of Medicine.

\section{Conflict of Interest}

The authors state that they have no Conflict of Interest (COI).

\section{References}

[1] Rademakers, R., Baker, M., Nicholson, A.M., Rutherford, N.J., Finch, N., Soto-Ortolaza, A., et al. (2011) Mutations in the Colony Stimulating Factor 1 Receptor $(C S F 1 R)$ Gene Cause Hereditary Diffuse Leukoencephalopathy with Spheroids. Nature Genetics, 44, 200-205. http://dx.doi.org/10.1038/ng.1027

[2] Stanley, E.R., Berg, K.L., Einstein, D.B., Lee, P.S. and Yeung, Y.G. (1994) The Biology and Action of Colony Stimulating Factor-1. Stem Cells, 12, 15-24.

[3] Pollard, J.W. (2009) Trophic Macrophages in Development and Disease. Nature Reviews Immunology, 9, $259-270$. http://dx.doi.org/10.1038/nri2528

[4] Kondo, Y., Kinoshita, M., Fukushima, K., Yoshida, K. and Ikeda, S. (2013) Early Involvement of the Corpus Callosum in a Patient with Hereditary Diffuse Leukoencephalopathy with Spheroids Carrying the de Novo K793T Mutation of CSF1R. Internal Medicine, 52, 503-506. http://dx.doi.org/10.2169/internalmedicine.52.8879

[5] Kinoshita, M., Kondo, Y., Yoshida, K., Fukushima, K., Hoshi, K., Ishizawa, K., et al. (2014) Corpus Callosum Atrophy in Patients with Hereditary Diffuse Leukoencephalopathy with Neuroaxonal Spheroids: An MRI-Based Study. Internal Medicine, 53, 21-27. http://dx.doi.org/10.2169/internalmedicine.53.0863

[6] Mantovani, A., Sica, A. and Locati, M. (2005) Macrophage Polarization Comes of Age. Immunity, 23, 344-346. 
http://dx.doi.org/10.1016/j.immuni.2005.10.001

[7] Benoit, M., Desnues, B. and Mege, J.L. (2008) Macrophage Polarization in Bacterial Infections. The Journal of Immunology, 181, 3733-3739. http://dx.doi.org/10.4049/jimmunol.181.6.3733

[8] Verreck, F.A., de Boer, T., Langenberg, D.M., Hoeve, M.A., Kramer, M., Vaisberg, E., et al. (2004) Human IL-23Producing Type 1 Macrophages Promote but IL-10-Producing Type 2 Macrophages Subvert Immunity to (Мусо) Bacteria. Proceedings of the National Academy of Sciences of the USA, 101, 4560-4565. http://dx.doi.org/10.1073/pnas.0400983101

[9] Pulford, K.A., Sipos, A., Cordell, J.L., Stross, W.P. and Mason, D.Y. (1990) Distribution of the CD68 Macrophage/ Myeloid Associated Antigen. International Immunology, 2, 973-980. http://dx.doi.org/10.1093/intimm/2.10.973

[10] Hulette, C.M., Downey, B.T. and Burger, P.C. (1992) Macrophage Markers in Diagnostic Neuropathology. The American Journal of Surgical Pathology, 16, 493-499. http://dx.doi.org/10.1097/00000478-199205000-00008

[11] Moestrup, S.K. and Møller, H.J. (2004) CD163: A Regulated Hemoglobin Scavenger Receptor with a Role in the Anti-Inflammatory Response. Annals of Medicine, 36, 347-354. http://dx.doi.org/10.1080/07853890410033171

[12] Komohara, Y., Ohnishi, K., Kuratsu, J. and Takeya, M. (2008) Possible Involvement of the M2 Anti-Inflammatory Macrophage Phenotype in Growth of Human Gliomas. The Journal of Pathology, 216, 15-24. http://dx.doi.org/10.1002/path.2370

[13] Schaer, D.J., Schaer, C.A., Schoedon, G., Imhof, A. and Kurrer, M.O. (2006) Hemophagocytic Macrophages Constitute a Major Compartment of Heme Oxygenase Expression in Sepsis. European Journal of Haematology, 77, 432-436. http://dx.doi.org/10.1111/j.1600-0609.2006.00730.x

[14] Schaer, D.J., Schleiffenbaum, B., Kurrer, M., Imhof, A., Bächli, E., Fehr, J., et al. (2005) Soluble Hemoglobin-Haptoglobin Scavenger Receptor CD163 as a Lineage-Specific Marker in the Reactive Hemophagocytic Syndrome. European Journal of Haematology, 74, 6-10. http://dx.doi.org/10.1111/j.1600-0609.2004.00318.x

[15] Canna, S.W., Costa-Reis, P., Bernal, W.E., Chu, N., Sullivan, K.E., Paessler, M.E., et al. (2014) Brief Report: Alternative Activation of Laser-Captured Murine Hemophagocytes. Arthritis \& Rheumatology, 66, 1666-1671. http://dx.doi.org/10.1002/art.38379

[16] Tomokiyo, R., Jinnouchi, K., Honda, M., Wada, Y., Hanada, N., Hiraoka, T., et al. (2002) Production, Characterization, and Interspecies Reactivities of Monoclonal Antibodies against Human Class A Macrophage Scavenger Receptors. Atherosclerosis, 161, 123-132. http://dx.doi.org/10.1016/S0021-9150(01)00624-4

[17] Kelley, J.L., Ozment, T.R., Li, C., Schweitzer, J.B. and Williams, D.L. (2014) Scavenger Receptor-A (CD204): A Two-Edged Sword in Health and Disease. Critical Reviews in Immunology, 34, 241-261. http://dx.doi.org/10.1615/CritRevImmunol.2014010267

[18] Wiktor-Jedrzejczak, W., Dzwigala, B., Szperl, M., Maruszynski, M., Urbanowska, E. and Szwech, P. (1996) Colony-Stimulating Factor 1-Dependent Resident Macrophages Play a Regulatory Role in Fighting Escherichia coli Fecal Peritonitis. Infection and Immunity, 64, 1577-1581.

[19] Ogiku, M., Kono, H., Ishii, K., Hosomura, N. and Fujii, H. (2011) Role of Macrophage Colony-Stimulating Factor in Polymicrobial Sepsis According to Studies Using Osteopetrotic (op/op) Mice. Journal of Surgical Research, 169, 106-116. http://dx.doi.org/10.1016/j.jss.2009.10.023

[20] Dai, X.M., Ryan, G.R., Hapel, A.J., Dominguez, M.G., Russell, R.G., Kapp, S., et al. (2002) Targeted Disruption of the Mouse Colony-Stimulating Factor 1 Receptor Gene Results in Osteopetrosis, Mononuclear Phagocyte Deficiency, Increased Primitive Progenitor Cell Frequencies, and Reproductive Defects. Blood, 99, 111-120. http://dx.doi.org/10.1182/blood.V99.1.111

[21] Ghia, J.E., Galeazzi, F., Ford, D.C., Hogaboam, C.M., Vallance, B.A. and Collins, S. (2008) Role of M-CSF-Dependent Macrophages in Colitis Is Driven by the Nature of the Inflammatory Stimulus. American Journal of Physiology: Gastrointestinal and Liver Physiology, 294, G770-G777. http://dx.doi.org/10.1152/ajpgi.00453.2007

[22] Konno, T., Tada, M., Tada, M., Koyama, A., Nozaki, H., Harigaya, Y., et al. (2013) Haploinsufficiency of CSF-1R and Clinicopathologic Characterization in Patients with HDLS. Neurology, 82, 139-148. http://dx.doi.org/10.1212/WNL.0000000000000046 